\title{
Developing an early-warning system for spotting at-risk students by using eBook interaction logs
}

\author{
Gökhan Akçapınar ${ }^{1,2^{*}}$ D, Mohammad Nehal Hasnine ${ }^{1}$, Rwitajit Majumdar ${ }^{1}$, Brendan Flanagan $^{1}$ and Hiroaki Ogata ${ }^{1}$
}

\author{
* Correspondence: \\ akcapinar.gokhan.2m@kyoto-u.ac.jp \\ ${ }^{1}$ Academic Center for Computing \\ and Media Studies, Kyoto University, \\ Kyoto, Japan \\ ${ }^{2}$ Department of Computer \\ Education and Instructional \\ Technology, Hacettepe University, \\ Ankara, Turkey
}

\begin{abstract}
Early prediction systems have already been applied successfully in various educational contexts. In this study, we investigated developing an early prediction system in the context of eBook-based teaching-learning and used students' eBook reading data to develop an early warning system for students at-risk of academic failure -students whose academic performance is low. To determine the best performing model and optimum time for possible interventions we created prediction models by using 13 prediction algorithms with the data from different weeks of the course. We also tested effects of data transformation on prediction models. 10-fold cross-validation was used for all prediction models. Accuracy and Kappa metrics were used to compare the performance of the models. Our results revealed that in a sixteen-week long course all models reached their highest performance with the data from the 15th week. On the other hand, starting from the 3rd week, the models classified low and high performing students with an accuracy of over $79 \%$. In terms of algorithms, Random Forest (RF) outperformed other algorithms when raw data were used, however, with the transformed data $\mathrm{J} 48$ algorithm performed better. When categorical data were used, Naive Bayes (NB) outperformed other algorithms. Results also indicated that models with transformed data performed lower than the models created using categorical data. However, models with categorical data showed similar performance with models with raw data. The implications of the results presented in this research were also discussed with respect to the field of Learning Analytics.
\end{abstract}

Keywords: Early-warning systems, At-risk students, Educational data mining, Learning analytics, Academic performance prediction

\section{Introduction}

Digital learning materials especially digital textbooks is a core part of modern education, and the adoption of digital textbooks in education is increasing. The benefits of using digital textbooks in education are mutual between teachers and students. With a digital textbook system, teachers can display their contents in an interactive way of deepening classes in an easier-to-understand manner. For instance, teachers can enlarge the most critical parts, upload appropriate audio/video materials to support contents, select narration of certain sentences, etc. On the other hand, for students, digital textbooks are like a well-arranged information terminal where individual's learning

(c) The Author(s). 2019 Open Access This article is distributed under the terms of the Creative Commons Attribution 4.0 International License (http://creativecommons.org/licenses/by/4.0/), which permits unrestricted use, distribution, and reproduction in any medium, provided you give appropriate credit to the original author(s) and the source, provide a link to the Creative Commons license, and indicate if changes were made. 
activities, including one's personality, abilities, interactivity, engage-ability, pro-activeness are stored. In addition to its advantages on students' learning, digital text readers are capable of recording interactions regarding students' reading behaviors. However, despite the increase in use, research analyzing students' interaction with digital textbooks is still limited.

Previous studies were evident that students' academic performance can be predicted by using e-book based log data (Daniel and Woody 2013; Junco and Clem 2015). However, any recognized studies were not found that are carried out to develop an early warning system by utilizing this data. In general, early warning systems are perdition models used to prevent expected failure at an early stage. In higher education, these models help teachers to detect drop-outs (Chung and Lee 2019) or possible academic failures (Macfadyen and Dawson 2010). Hence, in this paper, we aimed at developing an early warning system for identifying at-risk students by analyzing students' eBook interaction data. This study defines at-risk students whose academic performance is less than $50 \%$ of the class based on their in-class exercises (i.e., quiz) and final exam score. Consequently, we address the following main research question and three related research questions:

How to predict students' end-of-semester academic performance based on their interactions on an eBook application?

a. What is the best algorithm to predict student performance?

b. Does data transformation effect algorithms' prediction performance?

c. How early can a prediction model predict students' academic performance?

The rest of the paper is organized as follows: In the Background section, we summarize recent research on the educational early warning systems. In the Method section, we describe our data collection platform and data analysis processes. In the Result section, we present the results obtained, and in the final section, we draw our conclusions and point out the possible directions for future work.

\section{Background}

\section{Educational early warning systems}

In higher education, early warning systems are referred to as the prediction models capable of unfolding important information about students such as retention, drop-out, academic outcome, etc. at an early stage. Recent studies carried out on early warning system have provided evidence in favor of adopting various techniques as the means of detecting at-risk, drop-out or low performing students while a course is in progress. Villagrá-Arnedo et al. (2017) addressed the early prediction of possible school dropout as a serious problem in education, but a rather complex issue to resolve. Villagrá-Arnedo et al. (2017)'s study also argued that to improve students' academic performance, having knowledge on their actual progress and trying to predict their outcome at the earliest stages of the learning process can be extremely helpful to act early and cut off the problems at the root. To provide a meaningful guide to teachers and students, this study attempted to detect study trends and behavior patterns and to identify the causes of learning problems. To overcome this problem, a black box approach is used to 
develop prediction models. The accuracy of the model is tested based on 336 students' data those who are enrolled in a first-year university course, yield approximately $70 \%$ in the last weeks of the course. Their study also developed a set of graphical tools is to interpret the output information.

For identifying at-risk students early in the semester that used standards-based grading, Marbouti et al. (2016) developed predictive models using performance data. Six different prediction methods, namely Logistic Regression, Support Vector Machine, Decision Tree, Multi-layer Perceptron, Naïve Bayes Classifier, and k-Nearest Neighbor were used to identify at-risk students in their courses. This study reported that it might be possible to use Naïve Bayes Classifier to identify at-risk students with high accuracy during the semester for a class of having at least 120 students and less than $10 \%$ failure rate.

$\mathrm{Hu}$ et al. (2014), proposed an early warning system to detect at-risk students based on learning portfolios recorded in a learning management system. For this, various data-mining techniques including C4.5, LGR, and CART were applied on students' learning activity data of an online undergraduate course. The proposed early warning system consists of a data mining engine, a knowledge base, and an inference engine, responsible for producing early warning rules at different weeks of the course. This study also reported that, after spending a short learning period in the course, the early warning system could provide accurate forecasts based on students' learning portfolio data as the course of study progresses.

A study carried out by Costa et al. (2017), about how likely students are about to fail in introductory programming courses, applied educational data mining techniques to early predict. This study applied four data mining techniques (DecisionTree, Support Vector Machine, Neural Network, and Naive Bayes) on two independent datasets. One of which is a dataset from a distance education of 10 weeks long. Another was from an on-campus course of 16 weeks long. This study found that, in distance education courses, the decision tree technique is able to reach up to $82 \%$ effectiveness when students performed at least $50 \%$. On the contrary, for an on-campus course, the decision tree technique reaches the effectiveness of $79 \%$ when the students have performed at least $25 \%$. Based on this analysis, this study intended to provide educators and teachers with relevant information that will help them in early decision-making.

A recent study conducted by Howard et al. (2018) analyzed undergraduate students data to build prediction models for the early warning system. This study specifically investigated on the optimal time while a course is in progress to apply an early warning system. After analyzing a dataset that only contains continuous assessment and background information variables, this study yield that, the optimal time to implement an early warning system is after half-way through the course because 5-6 weeks (half-way) is the suitable time for students to make changes in their learning pattern while the prediction algorithms retained a reasonable accuracy.

A study carried out by Baker et al. (2015) analyzed students webtext activity data that are collected through Soomo Learning Environment to early predict of a student's success and failure. This study found that, those students who access the educational resources early, then continue accessing them throughout the early weeks of the course, and finally performed well in the formative activities are most likely to succeed in the course. These three indicators can be used in the early weeks to predict whether or not 
a student will fail or success. According to this study, logistic regression based combined model having 59.5\% accuracy can identify students those who are most likely to perform poorly.

In Massive Open Online Courses (MOOCs), low completion rates are considered to be the main problem. To overcome this problem, He et al. (2015) conducted a study aiming to accurate early identifying students who are at risk of not completing courses in MOOCs. This study analyzed Discrete Optimization Dataset launched by the University of Melbourne. Two transfer learning algorithms LR-SEQ and LR-SIM based on regularized logistic regression were developed to support this study. This study yields that LR-SIM-based logistic regression was promising for early prediction because it has exceptional AUC in the first few weeks of the course.

In 2015, Kuzilek et al. (2015) of the Open University, UK published a case study for predicting at-risk students as early as possible. In this study, demographic (static) data and student interactions with the Virtual Learning Environment (VLE) system were analyzed using a Bayesian approach. Four predictive models were built for weekly prediction of at-risk students enrolled in two introductory courses. To present the results of the predictive models, a prototype of an online dashboard was developed where weekly predictions of at-risk students are delivered to the instructors.

Researcher of the Open University carried out a further study on early prediction to identify at-risk students (Wolff et al. 2014). In this study, researchers aimed to determine the optimum time to make student intervention and to develop predictive models for early detection of at-risk students. For analysis, CART and Bayes models are employed for combining demographic data and data from the virtual learning environment. Four prediction models were built to produce a list of students ranked based on their risk level. Predictions information of at-risk students is calculated and updated in a dashboard on a weekly-basis to feedback educators.

Concerning academic performance and eBook usage, there is a study that analyzed students' eBook interaction data indicates that the course outcome is directly related to reading a textbook (Daniel and Woody 2013). Junco and Clem (2015) also found a relation between students' digital reading traces and their academic performance. In this paper, we investigated whether digital textbook usage data can be used to develop an early warning system for students at-risk of academic failure.

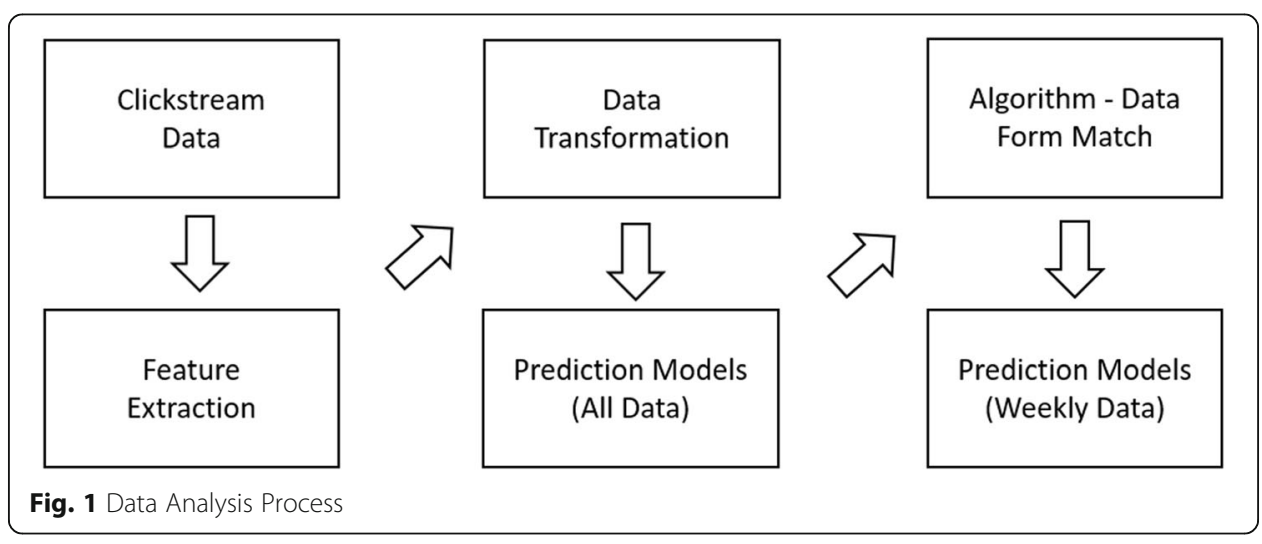




\section{Methodology}

We structured methodology and results section based on the process given in Fig. 1. We first collect clickstream data, then extract features related to students' reading behaviors. We prepared data in three different forms (raw, transformed, and categorical) to test data transformation effects on prediction algorithms. For data analysis, we first tested different prediction algorithms' performance on data obtained before the final exam. After that, we determined the best performing algorithm for each data form. Then, we tested these algorithms on data from different weeks of the course to see how early we can predict students' performances. Details of each section explained below.

\section{Participants and data collection}

For this study, data were collected from an eBook system (see Fig. 2.) named BookRoll that is developed by Ogata et al. (2015). The BookRoll system is currently being used in three universities in Asia. More than 10,000 university-students are using this eBook system to access their course materials inside or outside of the classroom. Fig. 2. shows the interface of the BookRoll system.

To elaborate more, the BookRoll system has several features including red and yellow markers to highlight important and difficult parts of a content, respectively. In the system, students can add memos for remembering important points. Also, the system lets students bookmark pages so that they can access them easily while they are reviewing the contents. Besides, the system has navigation functions such as next, previous, jump, etc. for navigating between different pages. At present, the BookRoll system works within the Learning Analytics framework that is proposed by (Flanagan and Ogata 2017). With the help of this framework, anonymized data regarding students' reading behaviors in the forms of Experience API (or xAPI) statements are collected. This

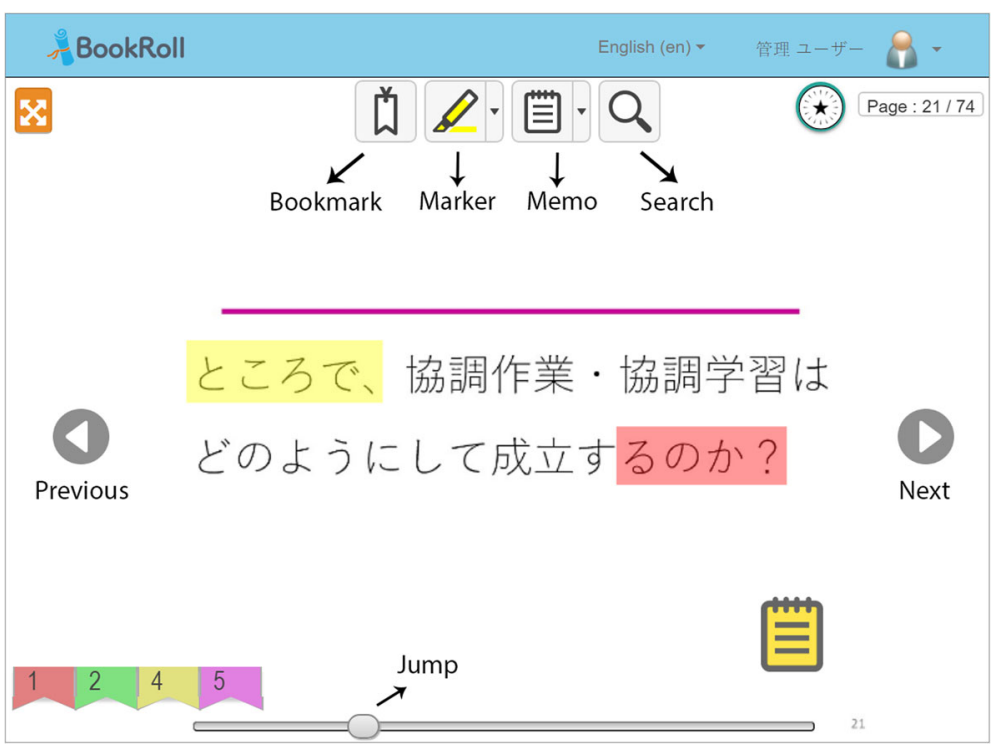

Fig. 2 BookRoll System 
framework, by ensuring students' privacy, helps to collect various kinds of interaction data that are related to their eBook reading behaviors.

The present study is based on the analysis of over 65,000 click-stream data that are collected from 90 students registered in an Elementary Informatics Course at a university. The course was offered to first-year undergraduate university students. Students used the eBook system to access the course materials that were uploaded by the instructor. The frequency of uploading the course materials by the instructor was once a week. Students were given 13 contents in different weeks of the course, and the eBook system recorded their interactions (e.g., next, previous, jump, highlight, adding a memo, bookmark, etc.). The semester was 16 weeks-long consisting of a 2-week-long break (i.e., 14 weeks for the whole course). Students' academic performance was evaluated based on eight exercises across the semester and one final exam. Students' performance calculated based on these exercises (40\%) and final exam score (60\%). Table 1 provides elaborated information more on the click-stream interaction data used for developing the early warning prediction system.

\section{Data preprocess}

The first step to our analysis was data preprocessing where we preprocessed the raw data and extracted various features which are reflected to students' reading interactions. Raw data were preprocessed in four steps. In the first step, we extracted features from the raw data collected in the database. In the second step, we analyzed descriptive statistics of features. Then, eliminated those features where students' usage was limited. Table 2 shows the features used in the prediction models.

In the third step, we created weekly data. The reason we created weekly data is to compare developed models' performances in different datasets to find optimum time for further interventions. In the fourth step, we applied data transformation and discretization methods to create three different data forms for each week's interaction data. These three data forms are raw data, transformed data, and categorical data.

For raw data, we used aggregated data without any modification. To prepare transformed data, we used percentile rank transformation to convert raw data between 0 and 1 based on the formula given below (1) where $P R$ is the percentile

Table 1 Number of logs in each event

\begin{tabular}{ll}
\hline Event Type & Number of Logs \\
\hline open & 2121 \\
next & 46,176 \\
previous & 17,025 \\
jump & 470 \\
redmarker & 51 \\
yellowmarker & 82 \\
memo & 416 \\
bookmark & 93 \\
other & 1491 \\
total & 67,925 \\
\hline
\end{tabular}


Table 2 Description of features used in prediction models

\begin{tabular}{ll}
\hline Features & Description \\
\hline totalevent & Total number of events \\
content & Number of different contents studied by the student \\
session & Number of reading sessions by the student \\
time & Total time spend on eBook system in minutes \\
week & Number of different weeks that student use the system \\
day & Number of different days that student use the system \\
completionrate & Average completion rate of all books \\
longevent & Number of events longer than $3 \mathrm{~s}$ \\
shortevent & Number of events less than or equal to $3 \mathrm{~s}$ \\
next & Number of Next events \\
previous & Number of Previous events \\
jump & Number of Jump events \\
redmarker & Number of red markers added by the student \\
yellowmarker & Number of yellow markers added by the student \\
memo & Number of memos added by the student \\
bookmark & Number of bookmarks added by the student \\
score & Academic performance of students at the end of the semester \\
\hline
\end{tabular}

rank score for each student, $f_{b}$ is the number of values which are less than the single student's value of the percentile rank, $f_{w}$ is the number of values which have the same value as the value of the single student percentile rank, and $N$ is the number of values.

$$
P R=\frac{f_{b}+1 /{ }_{2} f_{w}}{N}
$$

For categorical data, we discretized transformed values for all features into three categories as Low, Medium, and High by using equal width discretization method. Equal width discretization divides data into $\mathrm{k}$ intervals of equal size. We used previously transformed data and discretized each feature into three categories: Low (Score $<=$ 0.33), Medium ( $0.33<$ Score $<=0.66$ ), and High (Score $>0.66$ ).

The main reason to create three different data forms is to test the effects of data transformation on prediction performance following the research question. In terms of academic performance, we divided students into two groups: Low Performers (LP) and High Performers (HP). To do that, we first calculated the percentile rank of students based on their final scores and then labeled them as Low Performers (Score $<=0.5$ ) and High Performers (Score > 0.5).

\section{Data analysis}

In machine learning field, researchers generally test a combination of different algorithms, different feature sets, and different data preprocessing methods on their dataset to find the best performing model (Marbouti et al. 2016; Pandey and Taruna 2016; Romero et al. 2013). In this research, we followed a similar approach and tested different algorithms' performance on different data forms to find the best prediction model. 
We selected and compared performance of following algorithms based on previous research findings: AdaBoost, Bayesian Additive Regression Trees (BART), Stochastic Gradient Boosting (GBM), Generalized Linear Model (GLM), C4.5-like Trees (J48), Rule-Based Classifier (JRip), k-Nearest Neighbors (kNN), Naïve Bayes (NB), Neural Network (NNET), Random Forest (RF), Classification and Regression Tree (RPART), Support Vector Machines with Linear Kernel (SVMLinear), and eXtreme Gradient Boosting (xgbLinear).

During the data analysis, instead of comparing all algorithms with all data sets we followed the 2-steps analysis. In the first step, we aimed at finding the best-performed algorithm in each data form (e.g., Random Forest-Raw Data, etc.). For this, we took data from the database before the final exam week (we chose this week to eliminate the final exam effect from the data). Then we trained thirteen algorithms mentioned above by using three different data forms (i.e., raw, transformed, and categorical) to find the best-performed algorithm in each data form.

In the second step, we used the best-performed algorithm for each data form and tested its performance on the data obtained from different weeks of the course. In that case, we compared three algorithm-data form pairs' (e.g., Random Forest-Raw Data, etc.) performance in weekly data.

Each model's performance was evaluated by 10 -fold cross-validation. Since we have a balanced dataset, we used Classification Accuracy as the primary performance criteria to compare the results of the prediction models. We also used Cohen's Kappa as secondary metrics. It assesses the degree to which the model is better than chance at identifying the low and high performers. A Kappa of 0 indicates the chance level performance while a Kappa of 1 means the perfect performance (Pardos et al. 2013).

In this research, data analysis was conducted by $\mathrm{R}$ data mining software ( $\mathrm{R}$ Core Team 2017). We used dplry (Wickham et al. 2017) and arules (Hahsler et al. 2017), two $\mathrm{R}$ packages for data preprocessing. Machine learning analysis was conducted by using caret (Kuhn 2008) package.

\section{Results}

\section{Descriptive statistics and data preprocess}

Descriptive statistics of all features for each group and the results of the statistical difference test are shown in Table 3. Result yield that High Performers used BookRoll system more than Low Performers in terms of total time and the total number of actions. High Performers logged-in to the system more than Low Performers. They spent more time and did more interactions with the contents. Their average completion rates of the 13-contents were also significantly higher than Low Performers. With regard to jump, marker, memo, and bookmark, there was a significant difference observed between the two groups, but both groups' data were low, especially at the beginning of the semester. Hence, these features were excluded from the dataset.

As stated earlier, for data preprocessing, we created three different forms of data, namely raw, transformed, and categorical. Distribution of raw data and transformed data is displayed in Fig. 3. Since we used transformed data to create categorical data, distribution of students remained same in each category. 
Table 3 Descriptive statistics of features

\begin{tabular}{|c|c|c|c|c|c|}
\hline \multirow[t]{2}{*}{ Features } & \multicolumn{2}{|c|}{ High Performers $(n=45)$} & \multicolumn{2}{|c|}{ Low Performers $(n=45)$} & \multirow{2}{*}{$\begin{array}{l}t \text { Test } \\
\text { t-value }\end{array}$} \\
\hline & mean & SD & mean & SD & \\
\hline contentcount & 9.33 & 3.84 & 5.2 & 5.15 & $\mathrm{t}=4.31(p<0.001)$ \\
\hline sessioncount & 11.62 & 5.82 & 4.73 & 4.83 & $\mathrm{t}=6.11(p<0.001)$ \\
\hline totaltime & 320 & 288 & 93.44 & 109 & $\mathrm{t}=4.92(p<0.001)$ \\
\hline totalevent & 1068 & 1093 & 441 & 607 & $\mathrm{t}=3.36(p<0.01)$ \\
\hline uniqueweek & 6.24 & 2.47 & 2.58 & 2.58 & $\mathrm{t}=6.88(p<0.001)$ \\
\hline uniqueday & 7.44 & 3.30 & 3.02 & 2.97 & $\mathrm{t}=6.68(p<0.001)$ \\
\hline completionrate & 67.13 & 24.77 & 40.84 & 34.69 & $\mathrm{t}=4.13(p<0.001)$ \\
\hline longevent & 328 & 330 & 130 & 172 & $\mathrm{t}=3.57(p<0.001)$ \\
\hline shortevent & 739 & 789 & 311 & 456 & $\mathrm{t}=3.15(p<0.01)$ \\
\hline next & 719 & 694 & 307 & 425 & $\mathrm{t}=3.39(p<0.01)$ \\
\hline prev & 272 & 305 & 105 & 155 & $\mathrm{t}=3.26(p<0.01)$ \\
\hline open & 32.67 & 26.38 & 14.47 & 17.03 & $\mathrm{t}=3.88(p<0.001)$ \\
\hline jump* & 8.93 & 17.29 & 1.51 & 4.19 & $\mathrm{t}=2.79(p<0.01)$ \\
\hline marker* & 2.36 & 4.28 & 0.6 & 2.09 & $\mathrm{t}=2.47(p<0.05)$ \\
\hline memo* & 8.91 & 42.32 & 0.33 & 1.55 & $\mathrm{t}=1.36(p<0.5)$ \\
\hline bookmark* & 0.44 & 0.72 & 3.42 & 15.26 & $\mathrm{t}=-1.3(p<0.5)$ \\
\hline score & 84.58 & 11.25 & 47.71 & 19.72 & $\mathrm{t}=10.8(p<0.001)$ \\
\hline
\end{tabular}

*Features that were excluded from the dataset because of students' low participation

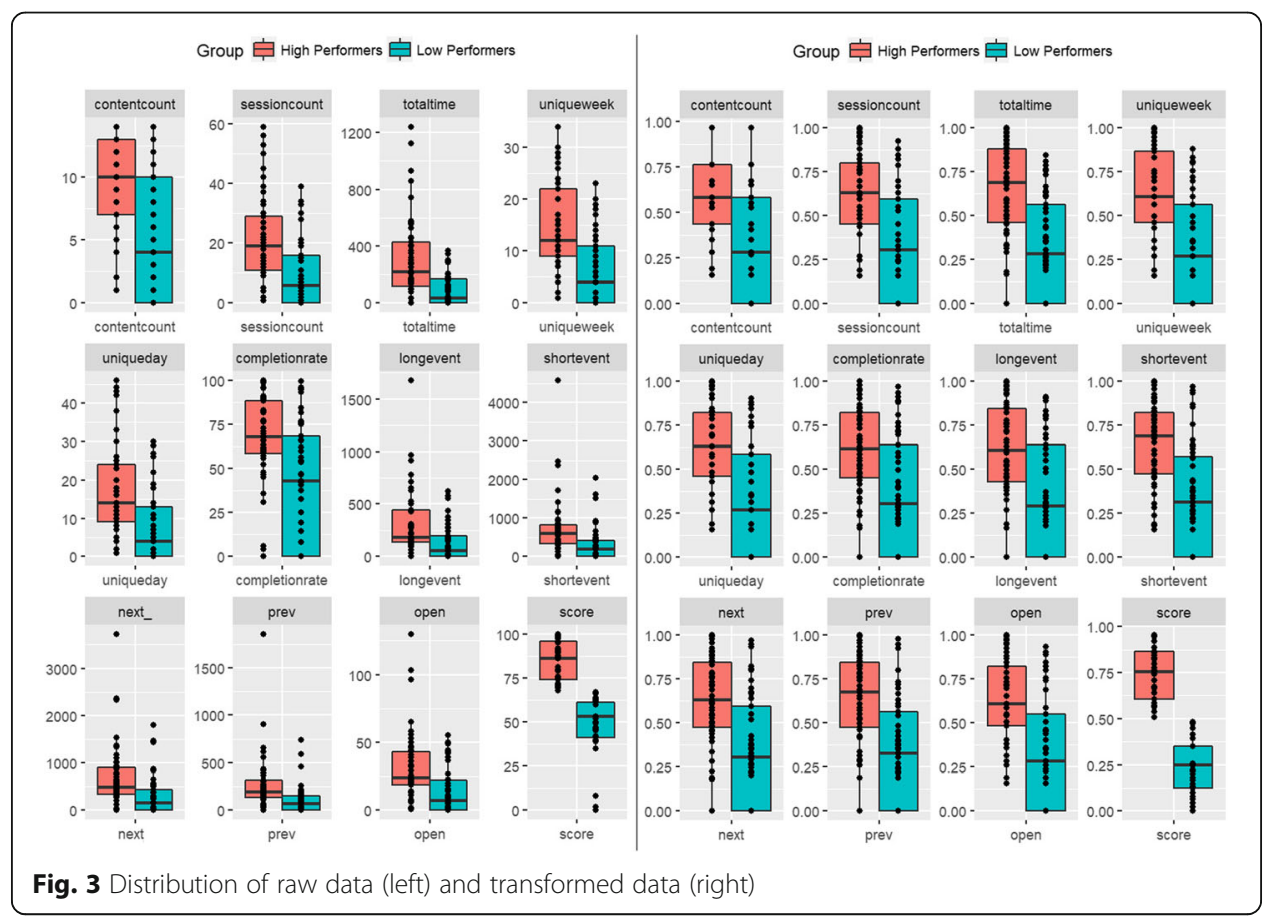




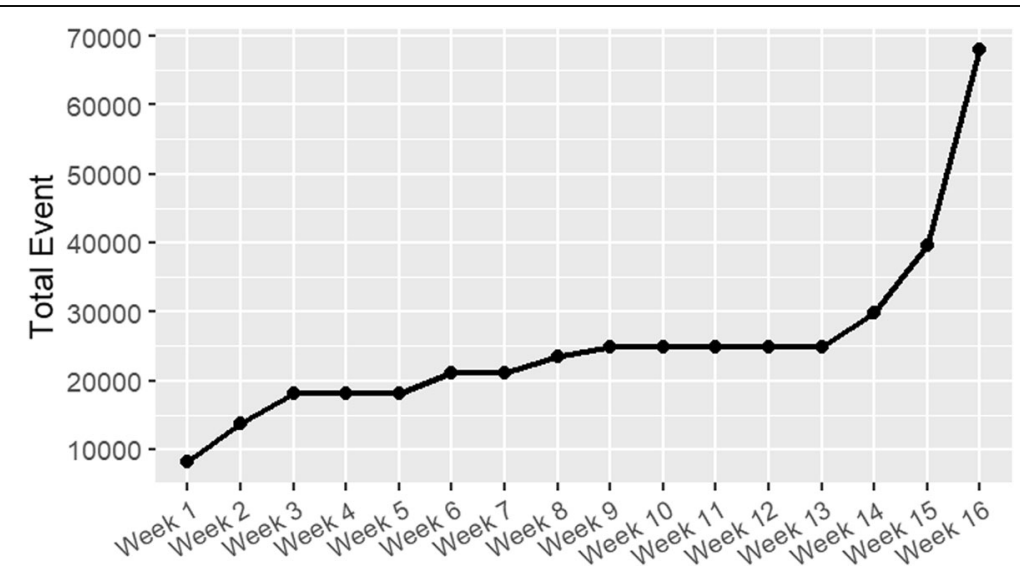

Fig. 4 Cumulative number of total events in each week

\section{Prediction models}

To create an early prediction model, we took data from different weeks of the course and compared the performance of the prediction algorithms on that datasets. Fig. 4 shows the cumulative number of events collected in the learning record store regarding students' reading interactions. A total number of events is 67,925 . The number of events recorded in the last two weeks (just before the final exam) was almost equal to the number of events recorded during the rest of the semester. In Fig. 4, it can also be seen that the number of events did not change between Week 3 and Week 5, Week 6 and Week 7, and Week 9 and Week 13. Therefore, we took data from Week 1, Week 2, Week 3, Week 6, Week 8, Week 9, Week 14, Week 15, and Week 16 to create the prediction models.

Table 4 Average scores of the models in terms of Accuracy and Kappa

\begin{tabular}{|c|c|c|c|c|c|c|}
\hline \multirow[t]{2}{*}{ Algorithm } & \multicolumn{2}{|l|}{ Raw Data } & \multicolumn{2}{|c|}{ Transformed Data } & \multicolumn{2}{|c|}{ Categorical Data } \\
\hline & Accuracy & Kappa & Accuracy & Kappa & Accuracy & Kappa \\
\hline Adaboost & 0.790 & 0.580 & 0.783 & 0.567 & 0.748 & 0.494 \\
\hline bartMachine & 0.811 & 0.620 & 0.813 & 0.625 & 0.792 & 0.583 \\
\hline $\mathrm{gbm}$ & 0.795 & 0.589 & 0.793 & 0.586 & 0.782 & 0.565 \\
\hline $\mathrm{glm}$ & 0.753 & 0.504 & 0.728 & 0.454 & 0.680 & 0.359 \\
\hline$J 48$ & 0.813 & 0.625 & 0.833 & 0.665 & 0.766 & 0.530 \\
\hline JRip & 0.795 & 0.587 & 0.782 & 0.564 & 0.755 & 0.509 \\
\hline knn & 0.813 & 0.627 & 0.798 & 0.595 & 0.805 & 0.611 \\
\hline naive_bayes & 0.823 & 0.646 & 0.801 & 0.601 & 0.811 & 0.621 \\
\hline nnet & 0.710 & 0.420 & 0.780 & 0.558 & 0.754 & 0.505 \\
\hline rf & 0.823 & 0.647 & 0.824 & 0.644 & 0.782 & 0.563 \\
\hline rpart & 0.752 & 0.501 & 0.759 & 0.516 & 0.727 & 0.454 \\
\hline svmLinear & 0.776 & 0.550 & 0.749 & 0.498 & 0.684 & 0.369 \\
\hline xgblinear & 0.798 & 0.596 & 0.780 & 0.560 & 0.726 & 0.452 \\
\hline
\end{tabular}

Note: Bold values show the top three best-performed algorithms' results for each data form 


\section{Prediction models with all data}

We tested all 13 algorithms' performance on the data taken from the 14th week of the course (before the final exam). Table 4 reports the results of the analysis in terms of average Accuracy and Kappa obtained from crossvalidation.

For the raw data, RF outperformed other algorithms in terms of Accuracy and Kappa metrics. However, with the transformed data J48 algorithm performed better. For categorical data, NB outperformed other algorithms. On the other hand, almost all algorithms predicted Low and High Performers correctly with an accuracy between $72 \%$ and 84\%. In terms of Kappa values, most of the model produced acceptable results (at least $50 \%$ better than chance).

Based on these results, we decided the best algorithm for each dataset and continued weekly analysis with selected algorithms. Finally, we used the RF for raw data, J48 for transformed data, and NB for categorical data.

\section{Prediction models with weekly data}

We compared selected algorithms' performance on data from different weeks of the course. Table 5 and Fig. 5 shows the results of the weekly analysis. Best performed algorithm's results for each week is highlighted in bold.

Results indicate that models with transformed data performed lower than the other two models. However, models with categorical data showed similar performance with models with raw data. Based on Accuracy and Kappa values we continued further analysis with the data from Week 3, Week 6, and Week 15. We included Week 3 and Week 6 since these weeks found optimal in previous research to make interventions (Howard et al. 2018). We selected Week15 as a benchmark to compare Week3 and Week6 models' performance. In Table 6, the average performance of the models after cross-validation is given in the forms of the confusion matrix. Columns in Table 6 represent the actual data while rows are predicted values. We omitted the results of the models created with transformed data because of their low performance (see Table 5).

In Table 6, we can see that in Week 3, RF algorithm's average accuracy is $79.1 \%$. And this algorithm classified $40 \%$ of the students as High Performers while $39.1 \%$ as Low

Table 5 Average scores of the models in terms of Accuracy and Kappa

\begin{tabular}{|c|c|c|c|c|c|c|}
\hline \multirow[b]{2}{*}{ Week } & \multicolumn{2}{|c|}{ Raw Data - RF } & \multicolumn{2}{|c|}{ Transformed Data - J48 } & \multicolumn{2}{|c|}{ Categorical Data - NB } \\
\hline & Accuracy & Kappa & Accuracy & Kappa & Accuracy & Kappa \\
\hline W1 & 0.735 & 0.466 & 0.674 & 0.345 & 0.725 & 0.446 \\
\hline W2 & 0.737 & 0.475 & 0.705 & 0.412 & 0.720 & 0.443 \\
\hline W3 & 0.790 & 0.580 & 0.718 & 0.435 & 0.775 & 0.548 \\
\hline W6 & 0.803 & 0.606 & 0.778 & 0.554 & 0.809 & 0.617 \\
\hline W8 & 0.801 & 0.600 & 0.711 & 0.416 & 0.796 & 0.590 \\
\hline W9 & 0.798 & 0.596 & 0.739 & 0.478 & 0.819 & 0.637 \\
\hline W14 & 0.826 & 0.651 & 0.818 & 0.635 & 0.800 & 0.598 \\
\hline W15 & 0.840 & 0.679 & 0.803 & 0.605 & 0.826 & 0.651 \\
\hline W16 & 0.777 & 0.551 & 0.697 & 0.394 & 0.718 & 0.438 \\
\hline
\end{tabular}

Note: Accuracy and Kappa values of the best-performing model for each week are in bold 


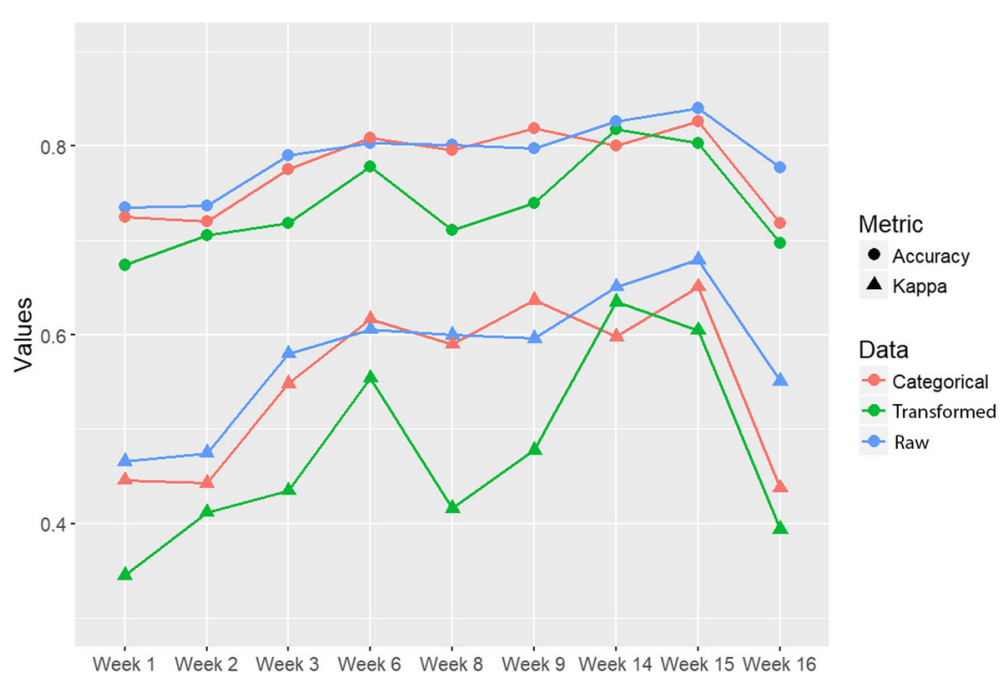

Fig. 5 Visualization of Accuracy and Kappa values

Performers. Ideally, $50 \%$ of the students should be High Performers $(n=45)$ and $50 \%$ should be Low Performers $(\mathrm{n}=45)$. Performance of the RF algorithm for the data from Week 6 is also similar, but it increased to $43.9 \%$ for High Performers and 42.1\% for Low Performers. On the other hand, for Week 3 data, NB algorithm with categorical data classified 36\% of the students as High Performers while $41.6 \%$ as Low Performers. For Week 6 data, the RF algorithm showed similar performance to Week 3, but NB algorithm predicted $64.5 \%$ of the students as Low Performers. For Week 15 data, both algorithms achieved their best performance. In general, RF predicted both classes equally. However, NB tends to predict Low Performers as High Performers, especially with the Week 3 and Week 6 data. Starting from Week 3 both algorithms predicted more than $80 \%$ of Low Performers correctly.

\section{Discussion and conclusion}

Low academic performance and high drop-out rates are important problems in education (Villagrá-Arnedo et al. 2017). On the other hand, studies showed that timely interventions

Table 6 Confusion matrix of the best perform models

\begin{tabular}{|c|c|c|c|c|c|c|c|}
\hline \multirow{2}{*}{$\overline{\text { Week }}$} & \multirow[b]{2}{*}{ Class } & \multicolumn{3}{|c|}{ Raw Data - RF } & \multicolumn{3}{|c|}{ Categorical Data - NB } \\
\hline & & $\mathrm{HP}$ & $L P$ & Total & $\mathrm{HP}$ & $L P$ & Total \\
\hline \multirow[t]{3}{*}{ W3 } & $\mathrm{HP}$ & $40.0 \%$ & $10.9 \%$ & $50.9 \%$ & $36.0 \%$ & $8.4 \%$ & $44.4 \%$ \\
\hline & LP & $10.0 \%$ & $39.1 \%$ & $49.1 \%$ & $14.0 \%$ & $41.6 \%$ & $55.6 \%$ \\
\hline & Total & $50 \%$ & $50 \%$ & $100 \%$ & $50 \%$ & $50 \%$ & $100 \%$ \\
\hline \multirow[t]{3}{*}{ W6 } & $\mathrm{HP}$ & $39.9 \%$ & $9.6 \%$ & $49.5 \%$ & $33.2 \%$ & $2.3 \%$ & $35.5 \%$ \\
\hline & LP & $10.1 \%$ & $40.4 \%$ & $50.5 \%$ & $16.8 \%$ & $47.7 \%$ & $64.5 \%$ \\
\hline & Total & $50 \%$ & $50 \%$ & $100 \%$ & $50 \%$ & $50 \%$ & $100 \%$ \\
\hline \multirow[t]{3}{*}{ W15 } & $\mathrm{HP}$ & $43.9 \%$ & $7.9 \%$ & $51.8 \%$ & $38.3 \%$ & $5.8 \%$ & $44.1 \%$ \\
\hline & LP & $8.1 \%$ & $42.1 \%$ & $50.2 \%$ & $11.7 \%$ & $44.2 \%$ & $55.9 \%$ \\
\hline & Total & $50 \%$ & $50 \%$ & $100 \%$ & $50 \%$ & $50 \%$ & $100 \%$ \\
\hline
\end{tabular}

HP High performers, LP Low Performers 
for at-risk students are effective to help them to change their behaviors (Arnold and Pistilli 2012; Tanes et al. 2011). Detection of at-risk students at an early stage of the course is crucial to design interventions. However, especially in large classrooms, this can be a challenging task for instructors without getting technological support.

In this study, we analyzed students' reading patterns to create an early warning system to identify at-risk students. One of the key contributions of this present work is to use eBook-based click-stream data for developing an early warning system that can spot at-risk students while the semester is in progress. For our analysis, we first tested thirteen different prediction algorithms' performance on data before the final exam. We prepared data in three different forms (raw, transformed, and categorical) and tested all algorithms with these data. After that, we determined the best algorithm-data form pair for each case. Then, we tested these algorithms on data from Week1, Week2, Week3, Week6, Week9, Week14, Week15, and Week16 of the course to see how early we can predict students' performances.

Our results revealed that low and high performer students could be correctly identified based on their eBook interaction from the 3rd week of the course. Among all, models created with the 15th week of data outperformed others. In that week, the model was built using raw data predicted Low and High Performer students with an accuracy of $84 \%$. The model's highest accuracy was noted on the 3rd week and 6th week is $79 \%$ and $81 \%$, respectively. Previous studies also revealed that data from the third week could be used for early prediction (He et al. 2015).

After the 15th week, we found that the accuracy and kappa values decreased almost the same level as at the beginning of the semester. One of the possible reasons for this is that the low performers might have studied harder before the final exam. Therefore, this week, their interaction increased but they got low scores from the final exam and classifiers could not predict these students accurately.

In this study, we transformed the data into three different forms and tested their effects on prediction models' performance. We found that, the models with transformed data produced the lowest performance in all datasets. On the other hand, models with categorical data showed better performance than models used transformed (continues) data only. These findings are important to creating generic models since the quantity of the data is different in different courses, but it is possible to split data into the same levels (low, medium, high). Researchers also suggest data discretization to generate models that are easy to interpret by non-expert users (Romero et al. 2008a; Romero et al. 2008b).

The present study has a few limitations. First, we assumed that all students used BookRoll as the main source for preparing the course. However, we could not ensure the fact that students have not downloaded or printed out the course materials although the system does not allow these operations. Second, we eliminated features such as Marker and Memo since the usage of these actions were done by limited number of students. However, these features are essential to understanding how actively students engaged with the learning material. In the future, we are planning to implement instructional strategies to encourage students to write a memo or use marker while reading.

Identification of at-risk students was the primary aim of this study. However, related concern is to design interventions to support those at-risk students. Studies showed that timely intervention is important to help at-risk students to improve their performance (Arnold and Pistilli 2012; Tanes et al. 2011). By having an intervention, we can 
help these students to change their reading behaviors and improve their overall success. Hence, in the future, we plan to implement this prediction model into our learning analytics dashboard so that instructors get feedback about their students' level of interaction and level of at-risk in a timely manner.

\section{Abbreviations}

BART: Bayesian Additive Regression Trees; GBM: Stochastic Gradient Boosting; GLM: Generalized Linear Model; J48: C4.5-like Trees; JRip: Rule-Based Classifier; kNN: k-Nearest Neighbors; NB: Naïve Bayes; NNET: Neural Network; RF: Random Forest; RPART: Classification and Regression Tree; SVMLinear: Support Vector Machines with Linear Kernel; xgbLinear: eXtreme Gradient Boosting

\section{Acknowledgements}

Not applicable.

\section{Funding}

This work was partly supported by JSPS Grant-in-Aid for Scientific Research (S) 16H06304, NEDO Special Innovation Program on Al and Big Data 18102059-0 and Hacettepe University Scientific Research Projects Coordination Center SBI-2017-16268.

\section{Availability of data and materials}

The datasets used and/or analyzed during the current study are available from the corresponding author on reasonable request.

\section{Authors' contributions}

GA drafted the initial manuscript and performed data analysis. $\mathrm{MNH}$ reviewed the literature. GA and MNH reviewed and revised the manuscript into its final shape. RM and BF provided insight and editing of the manuscript. $\mathrm{HO}$ provided supervision of the research. All authors read and approved the final manuscript.

\section{Competing interests}

The authors declare that they have no competing interests.

\section{Publisher's Note}

Springer Nature remains neutral with regard to jurisdictional claims in published maps and institutional affiliations.

Received: 11 February 2019 Accepted: 22 April 2019

Published online: 10 May 2019

\section{References}

K.E. Arnold, M.D. Pistilli, in Proceedings of the 2nd International Conference on Learning Analytics and Knowledge. Course signals at Purdue: using learning analytics to increase student success (ACM, Vancouver, 2012), pp. 267-270.

R.S. Baker, D. Lindrum, M.J. Lindrum, D. Perkowski, Analyzing Early At-Risk Factors in Higher Education e-Learning Courses. Paper presented at the 8th International Conference on Education Data Mining (Madrid, 2015)

J.Y. Chung, S. Lee, Dropout early warning systems for high school students using machine learning. Child Youth Serv. Rev. 96 346-353 (2019). https://doi.org/10.1016/j.childyouth.2018.11.030.

E.B. Costa, B. Fonseca, M.A. Santana, F.F. de Araújo, J. Rego, Evaluating the effectiveness of educational data mining techniques for early prediction of students' academic failure in introductory programming courses. Comput. Hum. Behav. 73, 247-256 (2017). https://doi.org/10.1016/j.chb.2017.01.047

D.B. Daniel, W.D. Woody, E-textbooks at what cost? Performance and use of electronic v. print texts. Comput. Educ. 62, 18-23 (2013). https://doi.org/10.1016/j.compedu.2012.10.016.

B. Flanagan, H. Ogata, Integration of learning analytics research and production systems while protecting privacy. Paper Presented at the 25th International Conference on Computers in Education (APSCE, Christchurch, 2017).

Hahsler, M., Buchta, C., Gruen, B., \& Hornik, K. (2017). arules: Mining Association Rules and Frequent Itemsets. Retrieved from https://CRAN.R-project.org/package=arules.

J. He, J. Bailey, B.I.P. Rubinstein, R. Zhang, in Proceedings of the Twenty-Ninth AAAl Conference on Artificial Intelligence. Identifying at-risk students in massive open online courses (ACM, Austin, 2015), pp. 1749-1755

E. Howard, M. Meehan, A. Parnell, Contrasting prediction methods for early warning systems at undergraduate level. Internet High. Educ. 37, 66-75 (2018). https://doi.org/10.1016/j.iheduc.2018.02.001.

Y.-H. Hu, C.-L. Lo, S.-P. Shih, Developing early warning systems to predict students' online learning performance. Comput. Hum. Behav 36, 469-478 (2014). https://doi.org/10.1016/j.chb.2014.04.002.

R. Junco, C. Clem, Predicting course outcomes with digital textbook usage data. Internet High. Educ. 27, 54-63 (2015). https://doi.org/10.1016/j.iheduc.2015.06.001.

M. Kuhn, Building predictive models in R using the caret package. J. Stat. Softw. 28(5), 1-26 (2008). https://doi.org/10.18637/ jss.v028.i05.

J. Kuzilek, M. Hlosta, D. Herrmannova, Z. Zdrahal, A. Wolff, LAK15 Case Study 1: OU Analyse: Analysing At-Risk Students at The Open University, Learning Analytics Review, no. LAK15-1, March 2015, ISSN: 2057-7494. http://www.laceproject.eu/learninganalytics-review/analysing-at-risk-students-at-open-university/

L.P. Macfadyen, S. Dawson, Mining LMS data to develop an "early warning system" for educators: A proof of concept. Comput. Educ. 54(2), 588-599 (2010). https://doi.org/10.1016/j.compedu.2009.09.008. 
F. Marbouti, H.A. Diefes-Dux, K. Madhavan, Models for early prediction of at-risk students in a course using standards-based grading. Comput. Educ. 103, 1-15 (2016). https://doi.org/10.1016/j.compedu.2016.09.005.

H. Ogata, C. Yin, M. Oi, F. Okubo, A. Shimada, K. Kojima, M. Yamada, E-Book-based learning analytics in university education. Paper presented at the International Conference on Computer in Education (APSCE, Hangzhou, 2015).

M. Pandey, S. Taruna, Towards the integration of multiple classifier pertaining to the Student's performance prediction. Perspect. Sci 8, 364-366 (2016). https://doi.org/10.1016/j.pisc.2016.04.076

Z.A. Pardos, R.S.J.D. Baker, M.O.C.Z.S. Pedro, S.M. Gowda, in Proceedings of the Third International Conference on Learning Analytics and Knowledge. Affective states and state tests: investigating how affect throughout the school year predicts end of year learning outcomes (ACM, Leuven, 2013), pp. 117-124.

R Core Team. (2017). R: A language and environment for statistical computing: R Foundation for Statistical Computing. Retrieved from https://www.R-project.org/.

C. Romero, M.-I. López, J.-M. Luna, S. Ventura, Predicting students' final performance from participation in on-line discussion forums. Comput. Educ. 68, 458-472 (2013). https://doi.org/10.1016/j.compedu.2013.06.009.

C. Romero, S. Ventura, P.G. Espejo, C. Hervás, Data Mining Algorithms to Classify Students. Paper presented at the Educational data mining 2008 (2008a).

C. Romero, S. Ventura, E. García, Data mining in course management systems: Moodle case study and tutorial. Comput. Educ. 51(1), 368-384 (2008b). https://doi.org/10.1016/j.compedu.2007.05.016

Z. Tanes, K.E. Arnold, A.S. King, M.A. Remnet, Using signals for appropriate feedback: Perceptions and practices. Comput. Educ. 57(4), 2414-2422 (2011). https://doi.org/10.1016/j.compedu.2011.05.016

C.J. Villagrá-Arnedo, F.J. Gallego-Durán, F. Llorens-Largo, P. Compañ-Rosique, R. Satorre-Cuerda, R. Molina-Carmona, Improving the expressiveness of black-box models for predicting student performance. Comput. Hum. Behav. 72, 621-631 (2017). https://doi.org/10.1016/..chb.2016.09.001.

Wickham, H., Francois, R., Henry, L., \& Müller, K. (2017). dplyr: A Grammar of Data Manipulation. Retrieved from https://CRAN.Rproject.org/package $=$ dplyr.

A. Wolff, Z. Zdrahal, D. Herrmannova, J. Kuzilek, M. Hlosta, Developing predictive models for early detection of at-risk students on distance learning modules. Paper presented at the machine learning and learning analytics workshop at the 4th international conference on learning analytics and knowledge (LAK14) (Indianapolis, 2014).

\section{Submit your manuscript to a SpringerOpen ${ }^{\circ}$ journal and benefit from:}

- Convenient online submission

- Rigorous peer review

- Open access: articles freely available online

High visibility within the field

- Retaining the copyright to your article

Submit your next manuscript at $\boldsymbol{\nabla}$ springeropen.com 